\title{
Compétences perçues du pays d'origine et qualité perçue des produits au Cameroun : cas des laptops et laits de toilette
}

\author{
Ahmed Moustapha Mfokeu \\ Henri Wamba \\ Université de Yaoundé II (Cameroun)
}

doi: 10.19044/esj.2017.v13n1p319 $\quad$ URL:http://dx.doi.org/10.19044/esj.2017.v13n1p319

\begin{abstract}
With the globalization of economies, we are witnessing a proliferation of products of different origins in the Cameroonian market. The objective of this Article is to assess the effect of perceived skills of the country of origin on the perceived quality of the products in some Cameroonian urban environment. As the methodological level, a field investigation has been carried out in order to collect the data from a sample of 784 consumers surveyed via the Internet (online form filling) and supplemented by a classical questionnaire (paper pencil) in the cities of Douala and Yaoundé. This article uses the model of structural equations to latent variables. Thus, the main results show a positive effect of the perceived competence of the country on the product's perceived quality by the common Cameroonian consumer living in urban area. The effect of the country of manufacture is more significant than that of the country of design. We also notice an increase of the country of design's effect on the perceived quality of products when the technological complexity of the products increases. Conversely, a reduction of the country of manufacture's effect on the perceived quality of the products is recorded when the technological complexity of the products increases. In this way, the moderating effect of the technological complexity of a product is thereby proven.
\end{abstract}

Keywords: Perceived competence, country of origin, the country of manufacture, countries of design, perceived quality. 


\section{Résumé}

Avec la globalisation des économies, on assiste à une multiplication des produits d'origines diverses sur le marché camerounais. L'objectif de cet article est d'évaluer l'effet des compétences perçues du pays d'origine sur la qualité perçue des produits en milieu urbain camerounais. Sur le plan méthodologique, une enquête de terrain a été effectuée. Les données ont été collectées auprès d'un échantillon de 784 consommateurs interrogés via Internet et complétées par un questionnaire «papier crayon» dans les villes de Douala et Yaoundé. Cet article a recours au modèle d'équations structurelles à variables latentes. Les principaux résultats révèlent un effet positif de la compétence perçue du pays sur la qualité perçue des produits par le consommateur camerounais en milieu urbain. L'effet du pays de fabrication est plus significatif que celui du pays de conception. On note également un accroissement de l'effet du pays de conception sur la qualité perçue des produits lorsque la complexité technologique des produits augmente. A contrario, une diminution de l'effet du pays de fabrication sur la qualité perçue des produits est enregistrée lorsque la complexité technologique des produits augmente. L'effet modérateur de la complexité technologique des produits est de ce fait avéré.

Mots-clés : Compétence perçue, pays d'origine, pays de fabrication, pays de conception, qualité perçue.

\section{Introduction}

Avec la création de l'Organisation Mondiale du Commerce (OMC) en 1995, l'unification du marché Européen et l'Accord du Libre Echange Nord Américain (ALENA) en 1994, l'évolution des marchés internationaux s'est accélérée avec un accroissement des produits dont le processus de fabrication est passé par plusieurs pays. Parmi les conséquences, on note également une accélération des importations et exportations entre pays.

Le rôle de l'origine nationale comme variable intervenant dans l'évaluation de la qualité du produit a été largement reconnu ${ }^{33}$. D'après Zeithaml (1988), la qualité perçue d'un produit peut être définie comme un jugement du consommateur de l'excellence ou de la supériorité globale du produit. La qualité perçue varie en fonction de la catégorie du produit (Chamhuri et Batt, 2015) ou de la complexité technologique du produit.

${ }^{33}$ Chao, 1993 ; 1999, Kaissouni, 2014; Ahmed et d'Astous, 2015. 
Il est important de noter que l'image du pays d'origine, couramment utilisée dans les recherches antérieures, n'est pas suffisante pour cerner tout l'effet du pays d'origine. Si la Corée du Sud a une bonne image globale et que ses appareils électroniques ou ses voitures ont une bonne qualité perçue dans plusieurs pays, ce n'est pas toujours le cas pour ses vêtements ou ses produits de beauté. Obermiller et Spangenberg (1989) ont constaté par exemple que le label «Made in Germany» améliore l'évaluation d'une voiture mais affecte négativement l'évaluation d'une robe comparativement au label «Made in Italy».

Il convient donc de rechercher la cohérence entre le pays d'origine et la catégorie de produits concernée.

La notion de compétence ou capacité perçue semble également importante pour cerner l'effet du pays d'origine. C'est dans ce sens que Roth et Romeo (1992) ont utilisé le concept de «match» entre l'image du pays et le produit. Dans la même direction, Hamzaoui et Merunka (2005), parlent du «fit image globale du pays/produit» pour désigner la compétence perçue.

Il est évident que les responsables de marketing international s'intéressent grandement aux moyens à mettre en œuvre pour faciliter l'acceptation par le marché de produits évalués négativement à cause de la mauvaise compétence perçue du pays de conception ou de fabrication. La revue de la littérature nous a convaincus que le développement de stratégies de marketing appropriées pour atteindre cet objectif nécessite une méthode de recherche qui considère l'effet du pays d'origine en fonction du degré de complexité technologique des produits.

La question spécifique qui guide cette étude est celle de savoir quel est l'effet de la compétence perçue du pays d'origine sur la qualité perçue des produits ? Dès lors, l'objectif assigné à cette étude est de déterminer l'effet de la compétence perçue du pays sur la qualité perçue des produits au Cameroun. Usunier (2002) à mené une étude en France en utilisant les produits électroniques grand public et des appareils électroménagers. Kaissouni (2014) quant à lui s'est intéressé à une problématique semblable dans une étude portant sur le matériel agricole au Maroc. Les résultats de ces deux études sont cependant contradictoires. Alors que Kaissouni (2014) a trouvé que le pays d'origine avait un impact significatif sur la qualité perçue, Usunier (2002) est arrivé à des conclusions opposées. Notre étude a pour but de produire, dans le contexte camerounais, d'autres résultats susceptibles d'améliorer notre connaissance actuelle des effets du pays d'origine.

Afin d'élargir les travaux de Kaissouni (2014) et Usunier (2002), nous avons collecté les données dans les villes de Yaoundé et Douala pour une étude quantitative. Il s'agit de l'étude de l'effet du pays d'origine dans le contexte économique et culturel propre au Cameroun qui est souvent appelé «Afrique en miniature», en raison de sa diversité humaine, géographique, culturelle, 
linguistique et même comportementale (Tsapi et al., 2009), ce qui en fait presque une synthèse du continent Africain. De plus, nous avons considéré des produits qui varient en terme de complexité technologique. Le positionnement défendu par cette étude est que la compétence perçue du pays d'origine a un effet positif sur la qualité perçue des produits. Cet effet varie en fonction de la complexité technologique de la catégorie des produits.

Pour atteindre notre objectif de recherche, nous présentons successivement le cadre conceptuel, les hypothèses de recherche, la méthodologie adoptée, les résultats et leur discussion.

\section{Décomposition et regroupement du concept de pays d'origine}

Le contexte actuel de la globalisation des marchés est caractérisé par le phénomène d'éparpillement du système productif (Tsapi et al., 2009). Les travaux de Pharr (2005) montrent une décomposition du concept de pays d'origine suivant les étapes de la chaîne de production en pays de design, pays de fabrication, pays fournisseur de composants, pays d'assemblage, pays de la marque et pays des actionnaires. Cette décomposition rend complexe les travaux sur l'effet «pays d'origine».

Faisant face à la complexité des travaux sur l'effet «pays d'origine», un regroupement a conduit à la décomposition simplifiée du concept en d'une part, pays de design ou de conception, et, d'autre part, pays d'assemblage ou pays de fabrication (Ahmed et D'Astous, 1996; Chao, 1998; Insch et McBride, 1998; Ahmed et d'Astous, 2015). C'est cette décomposition simplifiée du concept de pays d'origine, en pays de conception et pays de fabrication, que nous avons adoptée dans cette recherche. Nous nous intéressons aux produits qui ont un pays de conception différent du pays de fabrication. On les appelle produits hybrides (Chao, 1993 ; Ettenson et Gaeth, 1991) ou produits binationaux (Hamzaoui-Essoussi et Merunka, 2005).

Par ailleurs, le consommateur fait face à un nombre infini de produits provenant souvent d'un même pays ou de produits semblables provenant de plusieurs pays. Il aura besoin des regroupements pour simplifier le mécanisme d'évaluation.

\section{Théorie de la catégorisation cognitive et pays d'origine}

La théorie de la catégorisation de Fiske et Pavelchak (1986) propose que l'individu cherche à catégoriser spontanément une personne ou un objet rencontré sur la base d'une appartenance catégorielle saillante, afin d'économiser les ressources cognitives, pour simplifier la tâche de compréhension.

Cette recherche fait appel à la théorie de la catégorisation cognitive pour mieux cerner le mode d'influence du concept du pays d'origine sur la qualité perçue des produits. Cette théorie stipule que les connaissances des 
individus sont organisées dans leur mémoire à long terme sous forme de catégories cognitives. Une catégorie cognitive existe dès que deux objets ou événements distincts sont traités de manière équivalente (Rosch, 1973 ; Mervis et Rosch, 1981). La catégorisation consiste en une comparaison entre un stimulus et un ensemble de connaissances catégorielles préexistantes et déjà organisées en mémoire (Cohen et Basu, 1987).

La catégorisation est une activité cognitive fondamentale, car, sans ce processus, les organismes ne pourraient interagir de manière profitable avec l'infinité d'objets et d'événements distincts qu'ils rencontrent (Mervis et Rosch, 1981). La fonction des catégories cognitives est de réduire la complexité de l'environnement et de le structurer en un nombre restreint de termes mémorisables et opérants (Ladwein, 1995). Sur la base de la théorie de la catégorisation cognitive, l'effet du pays d'origine sera cerné dans cette étude à travers la compétence perçue du pays à concevoir ou à fabriquer une catégorie de produits.

Nos hypothèses de recherche sont inspirées d'une analyse de la littérature existante, notamment sur le rôle de l'origine nationale comme variable intervenant dans l'évaluation de la qualité du produit (voir Kishnakumar, 1974; Bilkey et Nes, 1982 ; Cattin et al, 1982 ; Heslop, Liefeld et Wall, 1988 ; Chao, 1993 ; Liefeld, 1993 ; Peterson et Jolibert, 1995 ). Le prestige du pays correspond à une dimension évaluative importante pour les consommateurs. On peut donc s'attendre à ce que la valeur perçue d'un produit soit plus élevée lorsque le pays d'origine du produit est évalué positivement. De même, il est raisonnable de penser que l'évaluation des produits sera affectée par la compétence perçue du pays d'origine.

La discussion précédente nous amène à formuler les hypothèses de recherche suivante:

Hypothèses principale : La compétence perçue du pays d'origine a un effet positif sur la qualité perçue des produits au Cameroun.

En tenant compte de la décomposition du concept du pays d'origine en pays de conception et pays de fabrication, nous pouvons formuler les hypothèses secondaires suivantes :

$\mathrm{H}_{1}$ : La compétence perçue du pays de conception a un effet positif sur la qualité perçue des produits.

$\mathrm{H}_{2}$ : La compétence perçue du pays de fabrication a un effet positif sur la qualité perçue des produits.

\section{Compétence perçue du pays d'Origine et complexité technologique des produits}

Diverses études ont trouvé que la magnitude de l'effet du pays d'origine est modérée par diverses variables liées au produit, telles que la 
familiarité avec le produit (Insch et McBride, 2004), le degré d'implication envers le produit (Ahmed et $a l ., 1994$; Lee, et $a l ., 2005$ ), ou encore le degré de complexité technologique (Insch and McBride, 2004 ; Hamzaoui, 2009). L'influence du pays d'origine sur la qualité perçue existe d'autant plus que le produit est technologiquement complexe.

La sophistication technologique d'un produit augmente le risque et appelle à plus de méfiance (Okechuku, 1994 ; Cheron et Propeck, 1997). Le consommateur peut alors trouver refuge dans un pays synonyme de qualité pour certains produits spécifiques (l'Allemagne pour l'automobile par exemple). La provenance nationale d'un produit est une variable extrinsèque très importante que les acheteurs utilisent lorsqu'ils jugent la qualité d'un produit technologiquement complexe (Ahmed et d'Astous, 1996).

Si certaines études n'ont pu prouver l'existence de tels effets (comme l'étude conduite par Ettenson et al., 1988), c'est qu'elles n'ont pas porté sur des produits présentant une forte complexité technologique (Okechuku, 1994). Aussi pensons-nous que c'est parce que ces études sont réalisées dans les pays développés qui connaissent des standards de qualité pour les produits qui sont sur leurs marchés.

Il est nécessaire de déterminer dans quelle mesure le degré de complexité technologique d'un produit affecte positivement ou négativement l'influence du pays d'origine. Plus précisément, il s'agit de déterminer l'influence de la compétence perçue des pays de conception et de fabrication sur la qualité perçue du produit.

Des études récentes ont tenté de comparer l'importance relative du pays de d'assemblage et du pays de conception dans l'évaluation de produits (Insch et McBride, 2004), et de comprendre comment cette importance varie selon le degré de complexité technologique du produit (Ahmed et al., 2002a ; Ahmed et d'Astous, 2007).

Lorsque le produit est technologiquement plus complexe ou impliquant, le pays d'origine est plus important pour les consommateurs (Heslop, et al., 1987). Pour les produits hautement impliquants comme l'automobile, les consommateurs cherchent presque quatre fois plus l'information «pays d'origine» par rapport à des produits beaucoup moins impliquants comme les T-shirts (Hugstad et Durr, 1986).

Le niveau de complexité technologique que suscite l'évaluation d'un produit est une variable qui nous semble importante dans le cadre de cette étude.

La revue de la littérature nous permet ainsi de proposer les hypothèses suivantes relatives à la compétence perçue du pays d'origine en fonction du degré de complexité technologique de la catégorie de produit : 
$\mathrm{H}_{3}$ : L'influence de la compétence perçue du pays de conception sur la qualité perçue du produit augmente avec l'accroissement de la complexité technologique de la catégorie de produit.

$\mathrm{H}_{4}$ : L'influence de la compétence perçue du pays de fabrication sur la qualité perçue du produit diminue avec l'accroissement de la complexité technologique de la catégorie de produit.

Nous pouvons résumer les relations à tester dans le modèle suivant :

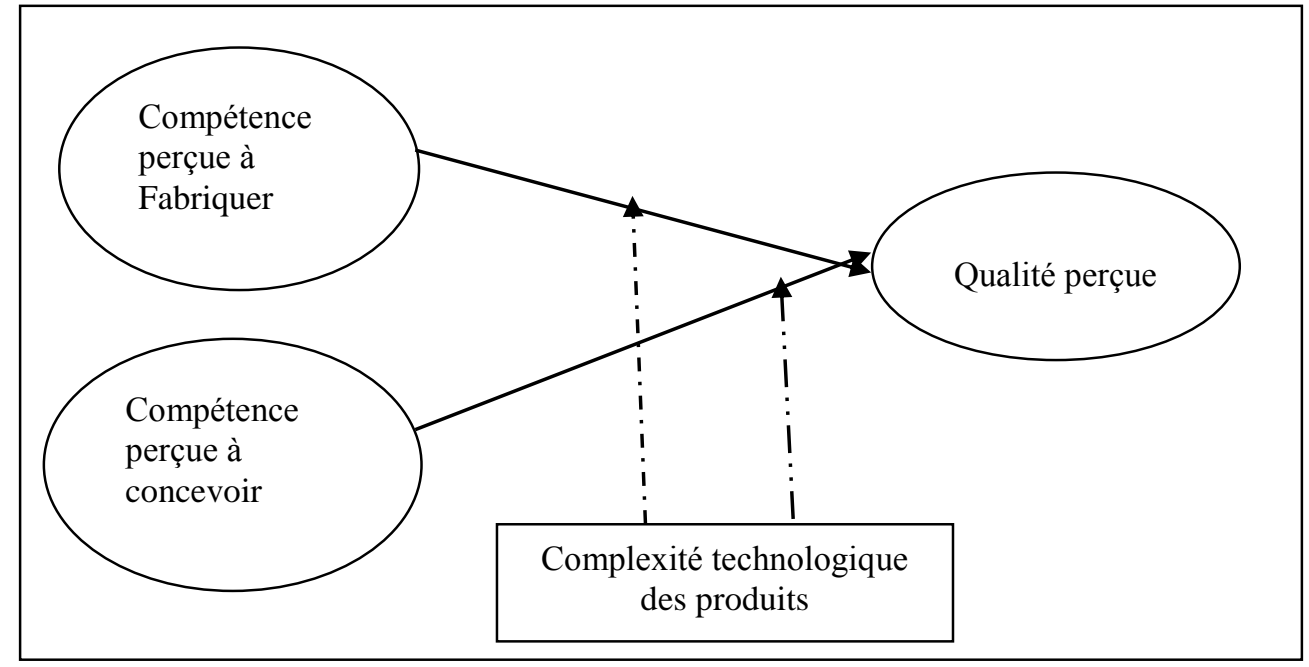

Figure 1 : modèle de l'effet de la compétence perçue du pays l'origine sur la qualité perçue

\section{METHODOLOGIE}

Une étude qualitative exploratoire a été menée auprès des consommateurs pour sélectionner les catégories de produits, les pays de conception et de fabrication nécessaires à la mise en place du design expérimental de cette recherche. C'est cette première étude exploratoire qui nous a permis de sélectionner les produits et les pays qui seront soumis par la suite à l'étude quantitative.

L'étude a porté sur deux catégories de produits : les laptops et les laits de toilette, correspondant à deux niveaux différents de complexité technologique. Huit pays ont été retenus sur la base de leurs différents niveaux de développement économique et capacité perçue à concevoir et à fabriquer les catégories de produits sélectionnés. Les pays retenus sont : la France, le Nigeria, la Chine, le Japon, les Etats-Unis, l'Italie, L'Allemagne et le Cameroun. Ces pays font partie des principaux partenaires économiques du Cameroun et les produits retenus dans cette étude sont pour la plupart issus de ces pays.

Le design de l'étude qui prend en compte les 3 variables (produit, pays de conception, pays de fabrication) est présenté dans le tableau suivant 
Tableau 1 - Design de l'étude

Produits Pays de Conception Pays de fabrication Echantillon

\begin{tabular}{llll}
\hline Laptop & USA & France & 405 \\
& & Chine & 405 \\
& Japon & Allemagne & 405 \\
Laits de toilette & NSA & France & 405 \\
& & Nigeria & 379 \\
& France & Italie & 379 \\
& & Cameroun & 379 \\
& & 379 \\
\hline
\end{tabular}

Nos hypothèses ont été testées auprès d'un échantillon de 784 consommateurs via une enquête sur Internet, complétée par une autre un questionnaire «papier-crayon». Nous avons opté pour une méthode d'échantillonnage non probabiliste. Ce choix se justifie par le fait qu'il n'existe pas de liste nominative et exhaustive des personnes qui consomment les produits étudiés.

La méthode utilisée est celle par quotas et les critères retenus sont l'âge et le sexe, vu leur pertinence prouvée dans la revue de la littérature sur des recherches similaires et la disponibilité des informations les concernant. Sur les 784 personnes ayant répondu de manière satisfaisante à cette enquête, $32,4 \%$ ont un âge qui varie entre 18 et 24 ans. $33 \%$ ont un âge qui varie entre 25 et 34 ans. $23,5 \%$ ont un âge qui varie entre 35 et 44 ans. $11,1 \%$ ont 45 ans et plus. Le tableau 2 ci-dessous montre la répartition de l'échantillon suivant l'âge.

Tableau 2- Répartition de l'échantillon par classe d'âge

\begin{tabular}{lll}
\hline Classe d'âge & Effectif & Fréquence (\%) \\
\hline $\mathbf{1 8}-\mathbf{2 4}$ ans & 254 & 32,4 \\
$\mathbf{2 5}$ - 34 ans & 259 & 33,0 \\
$\mathbf{3 5}$ - $\mathbf{4 4}$ ans & 184 & 23,5 \\
$\mathbf{4 5}$ ans et plus & 87 & 11,1 \\
Total & 784 & 100,0 \\
\hline
\end{tabular}

$56 \%$ personnes sont des hommes et $44 \%$ sont des femmes. Le tableau 3 ci dessous illustre la répartition des effectif en relation avec le sexe

Tableau 3-Répartition de l'échantillon par sexe

\begin{tabular}{lll}
\hline Genre & Effectif & Fréquence (\%) \\
\hline Masculin & 441 & 56,3 \\
Féminin & 343 & 43,8 \\
Total & 784 & 100,0 \\
\hline
\end{tabular}


La variable dépendante est la qualité perçue, les variables indépendantes sont la compétence perçue du pays de conception et la compétence perçue du pays de fabrication. Ces variables sont mesurées avec une batterie d'items grâce à une échelle de Likert à 7 points allant de «pas du tout d'accord» à «tout à fait d'accord».

Les évaluations des pays et des produits ont été précédées d'une mesure de la familiarité des répondants avec les catégories de produits étudiées à l'aide de la même échelle de Likert à 7 points (allant de «pas du tout d'accord» à «tout à fait d'accord»). Pour être admis à poursuivre le questionnaire sur les voitures ou les pantalons jeans, le degré de familiarité exigé devait correspondre à l'une des modalités suivantes : «Plutôt d'accord», «D'accord» ou «Tout à fait d'accord».

Le test de nos hypothèses est effectué par l'estimation d'un modèle d'équations structurelles.

Pour ce qui est du questionnaire «papier-crayon», la collecte des données sur le terrain a été suivie par l'opération de saisie des données. Cette opération a permis de transformer les informations sur papier en données, facilement accessibles à l'analyse. Nous avons utilisé CSPRO version 5 car il présente l'interface de saisie identique au questionnaire et les procédures de contrôles sont assez soignées.

Les données recueillies sur Internet ont été fusionnées avec celles du questionnaire «papier- crayon» pour avoir une base de données harmonisée. L'analyse des données s'est faite en deux étapes :

- Première étape : Statistiques descriptives

La première étape a consisté à présenter quelques statistiques descriptives mettant en exergue les différentes variables du modèle à partir du logiciel d'analyses Statistical Package for Social Science (SPSS 21). Par la suite, le test de comparaison des moyennes nous a permis de comparer les scores moyens des différents indicateurs. Les analyses factorielles confirmatoires (AFC) qui en ont suivi ont permis de construire les variables latentes de chacune des dimensions associées aux énoncés.

- Deuxième étape : Analyse économétrique

L'analyse économétrique effectuée grâce à un modèle d'équations structurelles a permis de mieux cerner les différentes corrélations entre les variables ainsi que leurs significativités. Les macros programmés à partir du logiciel XLSTAT ont permis de tester le modèle conceptuel de base.

\section{5.- RESULTATS}

\section{1. Evaluation de la compétence (capacité) perçue des pays d'origine}

Nous analyserons ici l'évaluation de la compétence perçue du pays de conception et du pays de fabrication sur la qualité perçue des produits. Le 
tableau 4 présente l'évaluation de la compétence perçue du pays de conception.

Tableau 4 - Evaluation de la compétence perçue des pays à concevoir (moyenne et écarttype)

\begin{tabular}{llll}
\hline Produits & Pays de conception & \multicolumn{2}{c}{ Capacité à concevoir } \\
& & Moyenne & Ecart-type \\
\hline $\begin{array}{l}\text { Ordinateurs } \\
\text { portables }\end{array}$ & USA & 6,24 & 1,42 \\
& Japon & 6,23 & 1,07 \\
Laits de toilette & USA & 5,86 & 1,23 \\
& France & 5,41 & 1,33 \\
\hline
\end{tabular}

D'après le consommateur camerounais en milieu urbain, les USA et le japon ont une compétence perçue semblable pour la conception des ordinateurs portables. Les USA ont une meilleure compétence perçue que la France pour la conception des laits de toilette.

Pour ce qui est de la compétence perçue à fabriquer les produits, les tendances peuvent être différentes de la compétence perçue à concevoir.

Le tableau 5 présente l'évaluation de la compétence perçue du pays de fabrication.

Tableau 5 - Evaluation de la compétence perçue des pays à fabriquer (moyenne et écarttype)

\section{Capacité à fabriquer le produit}

\begin{tabular}{llll}
\multirow{2}{*}{ Produits } & Pays de fabrication & Moyenne & Ecart-type \\
\hline Ordinateurs & Allemagne & 6 & 1,24 \\
portables & Chine & 5,87 & 1,29 \\
& France & 4,95 & 1,48 \\
& Nigeria & 3,44 & 1,62 \\
Laits de toilette & France & 5,53 & 1,23 \\
& Italie & 4,98 & 1,34 \\
& Nigeria & 3,92 & 1,47 \\
& Cameroun & 3,85 & 1,74 \\
\hline
\end{tabular}

Pour ce qui est des ordinateurs portables, l'Allemagne a la meilleure capacité perçue à fabriquer, avec une moyenne de 6 . Elle est suivie dans l'ordre décroissant par la Chine, la France et le Nigeria qui a une moyenne de 3,44 .

Pour ce qui est des laits de toilette, la France a la meilleure capacité perçue à fabriquer avec une moyenne de 5,53. Elle est suivie dans l'ordre 
décroissant par la l'Italie, le Nigeria et le Cameroun qui a une moyenne de 3,85 .

\subsection{Evaluation de la qualité perçue des produits}

La variable d'arrivée de notre étude est la qualité perçue des produits. L'évaluation de cette variable selon les pays de conception et de fabrication pour les quatre combinaisons, respectivement pour les ordinateurs portables et les laits de toilette, est présentée dans le tableau.

Tableau 6 - Evaluation de la qualité perçue des produits selon les pays de conception et de fabrication.

\begin{tabular}{|c|c|c|c|c|}
\hline Produits & Pays de conception & Pays de fabrication & Moyenne & Ecart-type \\
\hline \multirow{4}{*}{$\begin{array}{l}\text { Ordinateurs } \\
\text { portables }\end{array}$} & USA & France & 5,79 & 1,04 \\
\hline & & Chine & 5,58 & 1,07 \\
\hline & Japon & Nigeria & 4,32 & 1,27 \\
\hline & & Allemagne & 5,97 & 1,11 \\
\hline \multirow{4}{*}{$\begin{array}{l}\text { Laits } \\
\text { toilette }\end{array}$} & USA & France & 5,66 & 1,00 \\
\hline & & Nigeria & 4,21 & 1,21 \\
\hline & France & Italie & 5,49 & 1,04 \\
\hline & & Cameroun & 3,96 & 1,32 \\
\hline
\end{tabular}

La qualité perçue des Ordinateurs portables conçus aux USA varie selon que le pays de fabrication soit le France ou la Chine. Ceux qui sont fabriqués en France sont mieux notées que ceux qui sont fabriqués en Chine. Pour les Ordinateurs portables conçus au Japon, ceux qui sont fabriqués en Allemagne ont une qualité perçue meilleures que ceux qui sont fabriquées au Nigeria.

La qualité perçue des Laits de toilette conçus aux USA et fabriqués en France est meilleure que ceux qui sont conçus aux USA et fabriqués au Nigeria. Enfin, les laits de toilette conçus en France et fabriqués au Cameroun Italie sont moins bien perçus que ceux qui sont conçus en France et fabriqués en Italie.

En conclusion, Pour un même pays de conception, les produits fabriqués dans les anciens pays industrialisés (l'Allemagne, la France ou Italie) sont jugés de meilleures qualités que ceux fabriqués dans les pays comme la Chine, le Nigéria ou le Cameroun. Les laits de toilettes fabriqués au Cameroun ont la moins bonne notation malgré le fait que l'étude soit menée au Cameroun. 


\subsection{L'influence de la compétence perçue sur la qualité perçue des produits}

Le test des relations entre les variables d'entrée et la variable d'arrivée a été réalisé par l'estimation d'un modèle structurel reproduisant les relations supposées entre les construits latents. Le test est fait en considérant la compétence perçue du pays d'origine comme variable d'entrée, et la qualité perçue comme variable d'arrivée. Nous avons tenu compte de la compétence perçue du pays de conception et de la compétence perçue du pays de fabrication. Les résultats sont présentés dans le tableau 7.

Tableau 7 - Test de l'effet de la compétence perçue du pays d'origine à fabriquer ou à concevoir sur la qualité perçue des produits

\begin{tabular}{|c|c|c|c|c|c|}
\hline \multicolumn{6}{|c|}{ Equation de régression } \\
\hline \multicolumn{6}{|c|}{$\begin{array}{l}\text { Equation de régression : Y2 (qualité_produits) } \\
\text { Y2= } 2,203+0,388 \text { capacité_fabriquer }+0,176 \text { capacité_concevoir } \\
\left(p=0,000 ; R^{2}=0,315 R^{2} \text { ajusté=0,315) }\right.\end{array}$} \\
\hline & Valeur & $\begin{array}{l}\text { Erreur } \\
\text { standard }\end{array}$ & $\mathbf{T}$ & $\operatorname{Pr}>|t|$ & $\mathbf{f}^{2}$ \\
\hline Constante & 2,203 & 0,067 & 32,888 & 0,000 & \\
\hline capacité_fabriquer & 0,388 & 0,008 & 46,168 & 0,000 & 0,340 \\
\hline capacité_concevoir & 0,176 & 0,010 & 16,785 & 0,000 & 0,045 \\
\hline
\end{tabular}

Il apparait au vu de ce tableau que les coefficients associés à la compétence perçue du pays de conception et la compétence perçue du pays de fabrication sont positifs et significativement différents de zéro.

La compétence perçue du pays de conception a un effet positif sur la qualité perçue des produits. L'hypothèse $\mathrm{H} 1$ est ainsi validée.

La compétence perçue du pays de fabrication a un effet positif sur la qualité perçue des produits. L'hypothèse $\mathrm{H} 2$ est également validée.

Le résumé du modèle d'équation structurelle est présenté dans le graphique 1.

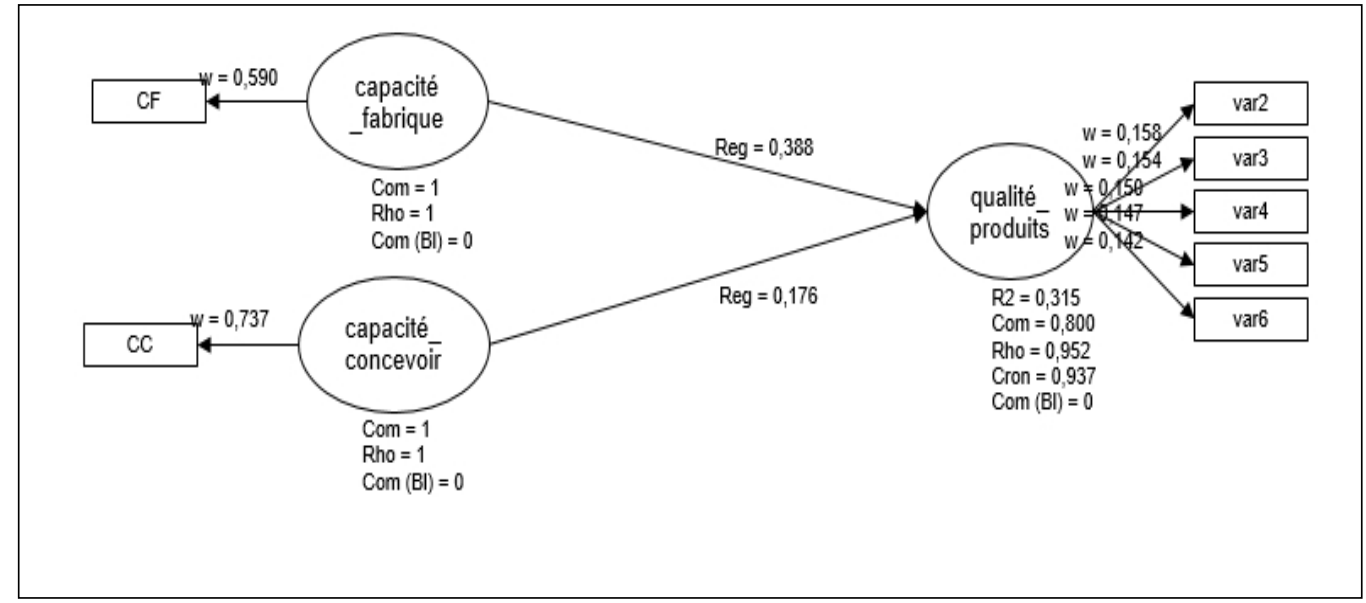

Graphique 1- Résume du modèle d'équation structurel du modèle 
La qualité perçue est une fonction de la capacité perçue du pays de conception et de la capacité perçue du pays de fabrication. Son équation de régression peut s'écrire de manière suivante : $Y 2=b+\alpha 2 \times 1+\beta 2 \times 2$

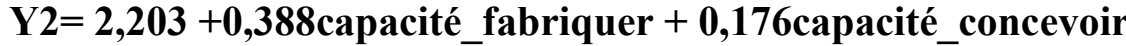

Où Y2 est la qualité perçue.

Les résultats obtenus permettent de faire le constat selon lequel : Le pays de fabrication a un effet plus important sur la qualité perçue que le pays de conception. Il ressort des résultats que, pour les voitures la compétence perçue du pays de fabrication a une influence plus importante que celle du pays de conception. De même, l'influence du pays de fabrication, à travers la compétence perçue à fabriquer, est plus importante que l'influence du pays de conception pour les pantalons jeans. Les consommateurs semblent donc accorder plus d'importance au pays de fabrication qu'au pays de conception pour toutes les catégories de produits étudiées.

En ce qui concerne la complexité technologique des produits ( $\mathrm{H} 3$ et H4), nous procédons au test de ces deux hypothèses en faisant une comparaison d'une part entre l'équation de régression de la qualité perçue des pantalons jeans (produit à faible complexité technologique) et d'autre part l'équation de régression de la qualité perçue des voitures (produits à forte complexité technologiques). Le tableau 8 montre le test l'influence de la compétence perçue des pays de conception et de fabrication sur la qualité perçue des produits en relation avec la complexité technologique.

Tableau 8 - Test de l'influence de la compétence perçue des pays de conception et de fabrication sur la qualité perçue des produits en relation avec la complexité technologique

\begin{tabular}{|c|c|c|c|c|c|c|c|c|}
\hline Régression & \multicolumn{8}{|c|}{ Equation de régression } \\
\hline++ & \multicolumn{8}{|c|}{$\begin{array}{l}\text { Equation de régression : Y (qualité_produits) } \\
Y 2 a=1,243+0,086 \text { capacité_concevoir }+0,255 \text { capacité_fabriquer+a } \\
\left(p=0,000 ; R^{2}=0,343 R^{2} \text { ajusté }=0,344\right)\end{array}$} \\
\hline \multirow{4}{*}{$\begin{array}{l}\text { produits de } \\
\text { complexité } \\
\text { technologique } \\
\text { faible }\end{array}$} & & Valeur & \multicolumn{2}{|c|}{\begin{tabular}{l|} 
Erreur \\
standar \\
d
\end{tabular}} & $\mathbf{T}$ & \multicolumn{2}{|c|}{$\operatorname{Pr}>|t|$} & $\mathbf{f}^{2}$ \\
\hline & Constante & 1,243 & \multicolumn{2}{|c|}{0,119} & $\begin{array}{l}10,43 \\
6\end{array}$ & \multicolumn{2}{|l|}{0,000} & \\
\hline & capacité_concevoir & 0,086 & \multicolumn{2}{|c|}{0,017} & 5,021 & \multicolumn{2}{|l|}{0,000} & 0,008 \\
\hline & capacité_fabriquer & 0,255 & \multicolumn{2}{|c|}{0,014} & $\begin{array}{l}18,38 \\
4\end{array}$ & \multicolumn{2}{|l|}{0,000} & 0,112 \\
\hline Régression & \multicolumn{8}{|c|}{ Equation de régression } \\
\hline \multirow{3}{*}{$\begin{array}{l}++ \\
\text { produits de } \\
\text { complexité } \\
\text { technologique } \\
\text { élevé }\end{array}$} & \multicolumn{8}{|c|}{$\begin{array}{l}\text { Equation de régression : Y (qualité_produits) } \\
\text { Y2b=0,946 }+0,143 \text { capacité_concevoir }+0,252 \text { capacité_fabriquer }+b \\
\left(p=0,000 ; R^{2}=0,411 R^{2} \text { ajusté }=0,410\right)\end{array}$} \\
\hline & & $\begin{array}{l}\text { Valeu } \\
\mathbf{r}\end{array}$ & $\begin{array}{l}\text { Erreur } \\
\text { standard }\end{array}$ & $\mathbf{T}$ & & $\begin{array}{l}\operatorname{Pr}> \\
|\mathbf{t}|\end{array}$ & \multicolumn{2}{|l|}{$\mathbf{f}^{2}$} \\
\hline & Constante & 0,946 & 0,109 & 8,6 & & 0,000 & & \\
\hline
\end{tabular}




\begin{tabular}{|l|l|l|l|l|l|}
\hline capacité_concevoir & 0,143 & 0,015 & 9,804 & 0,000 & 0,030 \\
\hline capacité_fabriquer & 0,252 & 0,013 & 19,165 & 0,000 & 0,114 \\
\hline
\end{tabular}

Dans ce tableau, une comparaison de nos coefficients de régression montre une augmentation des valeurs relatives à l'effet de la compétence perçue du pays de conception lorsque l'on passe des produits de complexité technologique faible aux produits de complexité technologiques élevés. Alors que l'on observe sous les mêmes conditions, une diminution des coefficients relatifs au pays de fabrication. L'hypothèse $\mathrm{H} 3$ est confirmée alors que l'hypothèse H4 est infirmée.

L'effet la compétence perçue du pays de conception sur la qualité perçue du produit augmente avec l'accroissement de la complexité technologique de la catégorie de produit, alors que l'effet la compétence perçue du pays de fabrication sur la qualité perçue du produit diminue avec l'accroissement de la complexité technologique de la catégorie de produit.

Nous récapitulons dans le tableau 9 ci-dessous les résultats obtenus lors des tests :

Tableau 9 - Récapitulatif des résultats du test des hypothèses.

\begin{tabular}{|l|l|l|l|}
\hline & HYPOTHESES & $\begin{array}{l}\text { RESULTATS DU } \\
\text { TEST }\end{array}$ & \\
\hline H1 & $\begin{array}{l}\text { La compétence perçue du pays de conception a un effet } \\
\text { positif sur la qualité perçue du produit }\end{array}$ & CONFIRMEE & \\
\hline H2 & $\begin{array}{l}\text { La compétence perçue du pays de fabrication a un effet } \\
\text { positif sur la qualite perçue du produit }\end{array}$ & CONFIRMEE & \\
\hline H3 & $\begin{array}{l}\text { L'effet de la compétence perçue du pays de conception sur } \\
\text { la qualité perçue du produit augmente avec l'accroissement } \\
\text { de la complexité technologique de la catégorie de produit. }\end{array}$ & CONFIRMEE & \\
\hline H4 & $\begin{array}{l}\text { L'effet de la compétence perçue du pays de fabrication sur } \\
\text { la qualité perçue du produit augmente avec l'accroissement } \\
\text { de la complexité technologique de la catégorie de produit. }\end{array}$ & INFIRMEE & \\
\hline
\end{tabular}

\section{6 - DISCUSSION}

Dans un premier temps, cette étude montre que la compétence perçue du pays de conception ou de fabrication a un effet positif sur la qualité perçue des produits ( Hypothèses $\mathrm{H} 1$ et $\mathrm{H} 2$ ). Les pays développés ont une meilleure compétence perçue que les pays moins développés pour les deux produits étudiés, les voitures et les pantalons jeans. Les consommateurs évaluent ainsi les produits en provenance des pays développés plus favorablement, en comparaison à ceux provenant des pays moins développés. Les résultats de notre étude vont dans le même sens que la plupart des études menées dans les pays en développement. (Wang et Lamb, 1983 ; Cordell, 1992). La métaanalyse de Verlegh et Steenkamp (1999) avait confirmé cette tendance. 
Dans les pays en développement, il existe une forte tendance à l'admiration des styles de vie des pays occidentaux chez les habitants, notamment chez les jeunes qui sont souvent ouverts à la nouveauté, à la modernité.

D'après Batra et al. (2000), l'admiration des styles de vie des pays développés a un effet significatif sur la préférence des produits en provenance de ces pays. De plus, les consommateurs ayant une grande susceptibilité à l'influence normative (les consommateurs sensibles à l'image sociale de soi) ont également une grande admiration envers les styles de vie des pays développés, car les produits en provenance de ces pays développés permettent de leur procurer un statut social élevé.

Cette préférence a été expliquée selon certains auteurs par la recherche d'un statut social élevé à travers la consommation de produits et de marques étrangères occidentales, synonymes de luxe, de modernité et de sophistication (Ger et al., 1993). Le choix des produits des pays développés permettrait également selon Venkatech et swamy (1994) aux consommateurs de pays émergents de sentir qu'ils ne sont pas exclus de la communauté internationale de consommation.

Par ailleurs l'explication suivant la théorie de la catégorisation est que le consommateur a tendance à catégoriser les produits importés d'un pays spécifique et au moment de l'évaluation de ce produit, cette catégorie sera récupérée à l'aide de l'attribut pays d'origine. D'après cette approche, le pays d'origine est considéré comme une catégorie cognitive constituée d'éléments que sont les différents produits conçus et/ou fabriqués dans ce pays (Odou et Nicholson, 1998). Ces études ont mis en évidence l'existence de catégories cognitives «pays d'origine» distinctes et selon lesquelles les consommateurs organisent les informations concernant les catégories de produits conçus et/ou fabriqués dans ce pays, ainsi que les caractéristiques qu'ils leurs associent. Ainsi, les catégories de produits ne sont pas attribuées de manière aléatoire aux pays, elles sont attribuées en fonction de la compétence perçue; par exemple, l'Allemagne est associée à l'électroménager et aux automobiles, le Japon au matériel électronique, etc.

La différence d'effet entre le pays de conception et de fabrication pourrait être expliquée de la façon suivante : les consommateurs accordent probablement plus d'importance à l'étape de fabrication d'un produit comparativement à celle de la conception (Hamzaoui-Essoussi , 2010). En effet, la fabrication repose sur des critères plus objectifs, plus tangibles alors que la conception tient compte des critères plus subjectifs, plus intangibles.

Ce résultat rejoint ceux de l'étude menée par Insch et McBride (2004), soulignant une plus grande importance accordée par les consommateurs de marchés émergents au pays de fabrication qu'au pays de conception.

Pour un tout autre type de produits (téléviseurs), Chao (1998) avait montré que le pays de design n'affectait qu'un aspect du produit, à savoir la 
qualité du design, alors que le pays de fabrication (assemblage) jouait sur la perception de la qualité globale d'un produit. Ahmed et al. (1997) étaient parvenus au même résultat auprès des consommateurs canadiens : en effet, selon eux, le pays de fabrication (assemblage) avait plus d'impact sur les évaluations des chaussures et des voitures que le pays de design.

Ce résultat va également dans le même sens que l'étude de Kaissouni (2014) qui a trouvé que sur le plan qualité, le consommateur a plus confiance plus dans les produits d'origine européenne notamment les pays de l'Europe de l'Ouest que dans les pays du reste du monde. L'étude de Kaissouni (2014) a porté sur le matériel agricole au Maroc.

Dans un deuxième temps cette étude montre que 1''effet de la compétence perçue du pays de conception sur la qualité perçue du produit augmente avec l'accroissement de la complexité technologique de la catégorie de produit, alors que 1'effet de la compétence perçue du pays de fabrication sur la qualité perçue du produit diminue avec l'accroissement de la complexité technologique de la catégorie de produit. (Hypothèses H3 et H4).

Ce résultat peut s'expliquer par le fait que le consommateur ne perçoit pas les produits technologiquement simples comme étant typique d'un pays particulier. C'est donc le pays de fabrication qui va jouer un rôle important. Ce résultat rejoint ceux de Hamzaoui-Essoussi (2010) qui a fait une étude en Tunisie et a trouvé que les consommateurs considèrent les fonctions de conception et de fabrication de manière différente selon le degré de complexité technique des produits. Ahmed et d'Astous (1015) ont également montré que la complexité technologique des produits modère les effets du pays d'origine dans un étude comparative menée entre le Canada et Taiwan.

\section{CONCLUSION ET IMPLICATIONS}

L'objectif de cet article était d'évaluer l'effet de la compétence perçue du pays d'origine sur la qualité perçue des produits. A l'aide des modèles d'équations structurelles à variables latentes, une estimation a été faite sur un échantillon de 784 consommateurs interrogés en ligne via Internet et par un questionnaire «papier crayon» dans les villes de Douala et Yaoundé. Les principaux résultats révèlent un effet positif de la compétence perçue du pays sur la qualité perçue des produits par le consommateur Camerounais en milieu urbain. L'effet du pays de fabrication est plus significatif que celui du pays de conception.

D'une part, la compétence perçue du pays d'origine est définie à un niveau global, ce qui nous a permis de distinguer la compétence perçue du pays de conception et la compétence perçue du pays de fabrication, et d'étudier leur influence respective sur la qualité perçue du produit selon son degré de complexité technologique. Ceci constitue un apport aux recherches sur 
l'influence du pays d'origine au niveau de la définition et de la compréhension du mode opératoire de cette influence à travers les deux composantes du pays d'origine, compte tenu du degré de complexité des produits et du marché étudié. Notre étude démontre que le rôle du pays d'origine dans l'évaluation des produits est un sujet d'étude complexe. Même si le pays d'origine est un attribut important, son influence sur les processus d'évaluation semble dépendre du type de produit considéré. De plus, le contexte de la recherche est le Cameroun, différent des contextes habituels de pays occidentaux des recherches sur l'effet du pays d'origine. Ce qui permet de confronter et de comparer les résultats.

D'autre part, les résultats de ce travail peuvent présenter un intérêt sur le plan managérial, en suggérant aux industriels et aux importateurs de réfléchir en termes de perception de la qualité de leurs produits par le consommateur camerounais. Ainsi, ils pourront prendre de meilleures décisions quant à l'origine nationale qu'ils souhaitent que le consommateur associe à leurs produits.

La marge de manœuvre de l'entreprise par rapport au pays de conception et au pays de fabrication est relativement importante. Lorsque le pays de conception ou de fabrication a une compétence perçue positive, l'entreprise pourra :

- proposer un nom reflétant le pays de conception ou de fabrication du produit ;

- mettre l'accent sur les attributs du produit influencés par l'image positive du pays de conception ou de fabrication du produit ;

- mettre en avant les informations concernant le pays de conception ou de fabrication du produit dans ses efforts de communication (publicité, promotion, etc.).

- Si le pays de conception ou de fabrication à une compétence perçue négative (du moins pour le produit en question), il sera nécessaire de contrecarrer cette image. Dans ce but, il est possible pour l'entreprise :

- de diminuer les informations se rapportant au pays de conception ou de fabrication du produit à la taille et à la visibilité minimales ;

- de localiser la production ou au moins l'assemblage, seule ou à travers des joint-

ventures, dans le pays préféré du consommateur en apposant par la suite le

label du pays de fabrication sur le produit ;

- de choisir un nom de marque rattaché à un pays à image globale ou une compétence perçue positive, quitte à tout ignorer de son identité nationale réelle. 
Cette étude présente plusieurs limites relatives tout d'abord à la prise en compte du pays de conception et du pays de fabrication sans fournir d'autres indices informationnels aux répondants.

Il serait pertinent de prendre en compte d'autres produits, de mener cette étude auprès de population d'autres pays en voies de développement afin de dégager les possibilités de généraliser les effets présentés ou de souligner la spécificité des résultats de notre étude aux marchés des pays en développement.

\section{References:}

1. Ahmed, S.A., d'Astous, A. and El Adraoui, M. (1994), "Country-oforigin effects on purchasing managers' product perceptions," Industrial Marketing Management, vol 23,no 4, pp. 323-32.

2. Ahmed, S.A. and d'Astous A. (1996), "Country-of-Origin and Brand Effects: A Multi-Dimensional and Multi-Attribute Study", Journal of International Consumer Marketing, vol. 9, no. 2, pp. 93-115.

3. Ahmed, S.A., d'Astous, A.and Lemire, S. (1997), "Country-of-origin effects in the U.S. and Canada: Implications for the marketing of products made in Mexico", Journal of International Consumer Marketing,10,1/2, pp. 73-92.

4. Ahmed, S.A., d'Astous, A. and Eljabri, J. (2002), "The impact of technological complexity on consumers" perceptions of products made in highly and newly industrialised countries", International Marketing Review, 19, 4/5, pp. 387-407.

5. Ahmed, S.A. and d'Astous, A. (2007), "French Canada and the Philippines: Comparing Product-Country Perceptions," Asia Pacific Management Review, vol12, no. 3, pp.133-143.

6. Ahmed, S. A. and d'Astous A. (2015), "Canada-Taiwan Differences in Product-Country Perceptions", International Journal of Commerce and Management, Vol. 25, no. 1, pp38-51.

7. Batra, R., Ramaswamy, V., Alden, D. L., Steenkamp, J.B., and Ramachander, S. (2000), "Effects of brand local and non-local origin on consumer attitudes in developing countries", Journal of Consumer Psychology, Vol.9, $\mathrm{N}^{\circ} 2$, pp 83-95.

8. Bilkey, W. J. and Nes, E. (1982), "Country-of-origin effects on product evaluations",Journal of International Business Studies, SpringSummer, pp. 89-99.

9. Cattin, P., olibert, A. and Lohnes, C. (1982), "A cross-cultural study of "made-in" concepts", Journal of International Business Studies, Winter, pp. 131-41.

10. Chao, P. (1993), "Partitioning country of origin effects: Consumer evaluations of a hybrid product", Journal of International Business Studies, vol. 24, no. 2, pp. 291-306. 
11. Chao, P. (1998), "Impact of country-of-origin dimensions on product quality and design quality perceptions", Journal of Business Research, vol. 42, no. 1, pp. 1-6.

12. Chamhuri, N and Batt, P.J. (2015), "Consumer perceptions of food quality in Malaysia“" British Food Journal, vol 117, no. 3, A1168, pp. 1168-1187.

13. Cheron, E., and Propeck, J. (1997), "The Effects of the Country-oforigin on the Evaluation of Products: A State of the Art Review and Research Propositions", IAE de Paris (University Paris 1. PantheonSoronne) - Gregor,7.

14. Cohen, J.B. and Basu, K. (1987), "Alternative models of categorisation: Toward a contingent processing framework", Journal of Consumer Research, 13, 4, pp. 455-72.

15. Cordell, V.V. (1992), "Effects of consumer preferences for foreign sourced products", Journal of International Business Studies, 23,2, pp. 251-259.

16. Ettenson, R. and Gaeth,G. (1991), "Consumer perceptions of hybrid (bi-national) products“, The Journal of Consumer Marketing, vol. 8, no. 4, pp. 13-8.

17. Ettenson, R., Wagner, J. \& Gaeth, G. (1988), "Evaluating the effect of country-of-origin and 'Made in the USA' campaign: A conjoint approach", Journal of Retailing, 64, 1, pp.85-100.

18. Fiske S.T. and Pavelchak M.A. (1986),"Category-based versus Piecemeal-based Affective responses: Development in SchemaTriggered Affect", Handbook of motivation and cognition,. Sorrentino R.M and Higgins T.E. (eds), New York: The Guiford, 162-173.

19. Hamzaoui-Essussi L. et Merunka D. (2005), “L'influence des pays de conception et pays de fabrication sur l'évaluation des produits binationaux", Cahier de Recherche, School of Management, University of Ottawa.

20. Hamzaoui-Essoussi, L. (2010), "Technological complexity and country-of-origin effects on binational product evaluation: investigation in an emerging market" Journal of Global Marketing, Vol. 23 no. 4. pp. 306-20.

21. Heslop, L.A., Liefeld J.P. and Wall M. (1987), "An Experimental Study of the Impact of COO Information", in Proceedings of the Annual Conference of the Administrative Sciences Association of Canada, R.Turner, editor.

22. Hugstad, P. S. and Durr, M. (1986), "A study of country of manufacturer impact on consumer perceptions", in Naresh Malhotra and John Hawes (ed.), Developments in Marketing Science, 9, Coral Gables, FL., Academy of Marketing Science, pp. 115-9. 
23. Insch, Gary S. and J. Brad McBride (1998), "Decomposing the country-of-origin construct: An empirical test of country of design, country of parts and country of assembl'“', Journal of International Consumer Marketing, vol. 10, no. 4, pp. 69-91.

24. Insch, G. S., McBride, J. B., (2004), "The impact of country-of-origin cues on consumer perceptions of product quality: A binational test of the decomposed country-of-origin construct", Journal of Business Research, 57,3, pp. 256-265.

25. Kaissouni H. (2014), “Les déterminants de choix d'un produit étranger et l'impact de l'originalité du produit dans le cadre des accords de libre échange sur le consommateur : cas du matériel agricole“, European Scientific Journal, vol.10, no.13, pp. 621-34.

26. Krishnakumar, P. (1974), "An exploratory study of the influence of country of origin on the product images of persons from selected countries“, PhD Dissertation, University of Florida.

27. Ladwein R. (1995), “Catégories cognitives et jugement de typicalité en comportement du consommateur",

28. Recherche et Applications en Marketing, 10(2), pp.89-100.

29. Liefeld, J. P. (1993), "Experiments on country-of-origin effects: Review and meta-analysis of effect size", in Papadopoulos, N. and L.A. Heslop (eds.), Product-Country Images: Impact and Role in International Marketing, International Business Press, New York, pp. 117-56.

30. Mervis, C.B. and Rosch E. (1981), "Categorization of natural objects", Annual Review of Psychology, 32, pp. 89-115.

31. Obermiller, C. and Spangenberg, E. R. (1989), "Exploring the effects of country-of-origin labels: An information processing framework", in Thomas K. Srull (ed.), Advances in Consumer Research, 16, pp. 45459.

32. Odou, P. and Nicholson, P. (1998), "L'influence des croyances stéréotypiques associées au pays d'origine d'un produit sur son évaluation : la mise en évidence du modèle catégoriel de traitement de l'information", Actes du XIVème Congrès de l'Association Française de Marketing, Bordeaux, 595-616.

33. Okechuku, C. (1994), "The importance of product country-of-origin: A conjoint analysis of the United States, Canada, Germany, and the Netherlands", European Journal of Marketing, 28, 4, pp. 5-19.

34. Peterson, R A. and Jolibert, A. (1995), "A meta-analysis of countryof-origin effects“, Journal of International Business Studies, vol. 26, no. 4, pp. 883-900. 
35. Pharr, J. M. (2005), "Synthesizing Country-of-Origin Research From the Last Decade: Is the Concept Still Salient in an Era of Global Brands? “', Journal of Marketing, Fall, pp. 34-45.

36. Rosch, E. (1973), "On the internal structure of perceptual and semantic categories", Cognitive development and the acquisition of language, éd. Moore, T. E., Academic Press, New York, 111-144.

37. Roth, M. S. And Romeo, J. B. (1992), "Matching product category and country image perceptions: A framework for managing country-oforigin effects", Journal of International Business Studies, vol. 23, no. 3, pp. 477-97.

38. Tsapi V., Djeumene P. and Tchuente M. (2009), "Rôle du pays d'origine dans la perception de la qualité du vin par le consommateur africain : une étude menée dans le contexte camerounais" Market Management Vol. 9 no 1 pp. 38-58.

39. Usunier, J-C.(2002), "Le pays d'origine influence-t-il encore les évaluations des consommateurs", Revue Française du Marketing, no. $189 / 190$, pp. 49-60

40. Venkatesh, A. and Swamy, S. (1994), "India as an emerging consumer society", A cultural analysis. In C. J. Schultz, II, R. W. Belk, \& G. Ger (Eds.), Research in consumer behavior (Vol. 7, pp. 193-223). Greenwich, CT: JAI.

41. Wang, C-K. and Lamb, C.W. Jr. (1983), "The impact of selected environmental forces upon consumers' willingness to buy foreign products", Journal of the Academy of Marketing Science, 11, 4, pp. 71-84.

42. Zeithaml, V. A. (1988), "Consumer perceptions of price, quality and value: A means-end model and synthesis of evidence", Journal of Marketing, vol. 52, July, pp. 2-22. 